\title{
Conf $-760209--4$
}

\section{Plasma-Wall Interaction in ATC During}

High Power Neutral Beam Injection*

by S. A. Cohen

Princeton University, Plasma Physics Laboratory Princeton, New Jersey 08540

\begin{abstract}
Measurements of the elemental composition of the vacuum vessel wall surface and impurity influx into ATC during high power beam-heated discharges are combined with previous measurements of power balance and scaling laws to give a self consistent model of plasma-wall interaction in ATC. It is shown that plasma charge exchange induced desorption is the main cause of impurity influx during neutral beam injection. Impurities charge the net power balance in these beam-heated discharges by $\sim 158$.
\end{abstract}

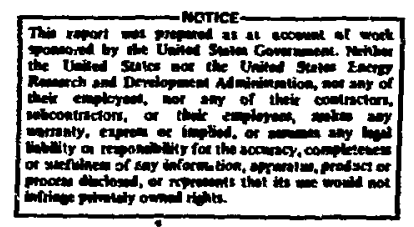


INTHODUCTION

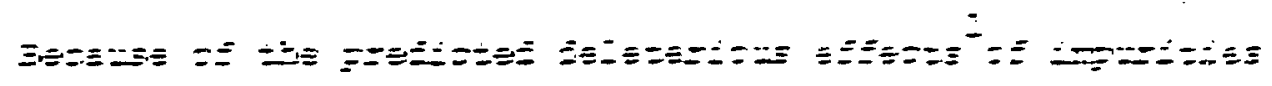

in future, large, beam-heated tokamaks it is importarit to ascertain the nature and extent of plasma wall interaction in present day. beam-heated tokamaks. Hence, when the density-confinement timetemperature parameters of $\mathrm{ATC}^{2}$ were extended to $2 \times 10^{14} \mathrm{~cm}^{-3}-.01$ sec - $1220 \mathrm{eV}$ by the combined use of high power neutral beams and adiabatic compression, measurements were made of the time behavior of selected impurities in the plasma and of changes in the elemental composition of the vacuum vessel wall inner surface? By combining these results with previous measurements of the power balance ${ }^{4}$ and empirical scaling laws we are able to form a selfconsistent model of the plasma-wall interactions in ATC. It is shown that the flux to the wall of charge exchange (CX) neutrals from the background plasma is the main cause of 0 (the dominant impurity in ATC) and C influx during the beam-heated portion of the discharge. Photodesorption by line radiation and $x$-rays, thermal desorption by runaway electrons, electron-stimulateddesorption by the cool, tenuous edge plasma, and sputtering by beam and plasma $C X$ neutrais cause smaller levels of impurity influx. We cannot make quantitative conclusions about the impurity influx due to the thermal load on the limiter. In these discharges, impurity effects improve the net plasma heating and containment by $-158^{\circ}$. 
PLASMA PARAMETERS

In the high power beam-heated, compressed ATC discharges ${ }^{2}$ a deuterium plasma is formed during the first few milliseconds and then resistively heated by an induced current of 60-80 kA. After $18 \mathrm{~ms}, 230 \mathrm{~kW}\left(15\right.$ amperes at 15 Volts) of neutral $D^{\circ}$ are injected as alsupplement to the/160 $\mathrm{kW}$ of Ohaic heating $(\mathrm{OH})$. The injectors are turned off after $10 \mathrm{~ms}$, and the plasma is adiabatically compressed by a factor of 2.1 in major radius and 1.45 in minor radius. Measured values of electron density and temperature and other parameters are shown in Table 1 for various times during a discharge. Three lines in Table 1 must receive special attention. The value of the central neutral density of $D^{\circ}$ shown in line 13 has been taken fror: a computer simulation ${ }^{6}$ of these neutral beam heated discharges. The results of this simulation (shown later in this section) are in reasonable agreement with the experimental measurements. The value of the electron density beyond the limiter near the vacuum vessel walls (line 11) was estimated from power balance arguments. Finally, it has previously been shown that $z_{\text {eff }}(1$ ine 15) is correlated with impurity concentrations and is not, in the main, a plasma resistance anomaly. In this particular case 0 contributes about 608 of the $z_{\text {eff }}$ value, and represents 38 of the plasma censity at $t=25 \mathrm{~ms}$.

In Figure 1 are displayed the electron and ion temperature and density profiles calculated by the code. The experimental measurements showed the central ion temperature to be 158 higher, the central electron temperature 108 lower and an electron density 
peaked on axis. The discrepancies in the temporatures are either due to - 108 errors in the code's heat transport coefficients or to a better than assumed coupling between ions and electrons. The peak in the electron density can be shifted on axis if the energy of the recycling $D^{\circ}$ is raised to (the more realistic value of) 100 $e V / D^{\circ}$ from the $10 \mathrm{eV}$ value used in this simulation.

The measured time evolution of the central ion temperature and the average electron density during the beam heated stage of the discharge are displayed in Figure 2. In addition, the measured energy losses through the surface of the plasma, i.e., cx out flux a. radiation, are shown. These latter measuxements ${ }^{1}$ were performed during $90 \mathrm{~kW}$ beam-heated discharges. In this set of experiments the absolute time dependent energy fluxes to the wall and to the limiter were measured. It was found that during the of portion of the discharge 80 of the energy outflow went to the limiter, and only 20 to the wall. (Energy loss to the limiter is via electron and ion thermal conduction and diffusion.) when beam heating was added to the OH the surface losses tripled due to three effects. The dominant effect is that 60 of the injected $15 \mathrm{keV}$ $D^{\circ}$ escape with their full energy from the plasma by $C x$. Secondly, the plasma ion temperature and central neutral density increase, leading to more numerous and more energetic escaping plasma $c x$ neutrals. Thixdly, there is generally an increase in the impurity influx level and radiation losses are correspondingly enhanced.

Figure 3 shows the time dependences of the energy input and loss terms calculated for the high power beam-heated run. The surface energy loss by $\mathrm{Cx}$ of beam ions is 10 times greater than by 
CX of plasma ions. However, the number of beam cX particles striking the wall to 10 times less than the number of plasm cx particles.

The calculated energy distribution of the total cx outflux is shown in Figure 4. Measurements down to 400 eV are in reasonable agreemant.

Earlier bremsstrahlung measurements on similar drischarges showed essentially a thermal character to the radiation. No evidence of runaway eloctons was secn.

The measured tine evolution of vacuum ultraviolet (vuV) radiation from selected Fe. $C$, $T i$ and $O$ ions is shown in Fjyure 5. ISeveral monolayers of ti had been sublimated over - half the surface of ATC $^{8}$ during tha hours immediately preceding there experIments.) For the Fe, $E$, and If (but not the o) data the plasma was compressed at 30 ms. Three time periods are maxhed on the vuy cata. During poriod 1 the plasma is being heated by the oll alone. Fhe clectron temperature is rapidly climbing. There is a rather uniform concentration of all iffurities seross the plasaat 2 These procted through higher ionization states as the temperature increases. This is the reason that the peaks in period 1 occur at different times for the different ions. During period 2 the photon signats show a plateau. This is indifative of a constant influx of each impurity. During period 3 the netutral beams are injected. Distinctly different behavior is seen in comparing the 0 (or c) signals with the Fe (or $T i$ ). Firstly, there is a small step-like increase at $t=18 \mathrm{~ms}$ for 0 . The 0 and $C$ signals then grow more rapidly than the electron donsity (cf. Figure 2). The Fe and $\mathrm{Ti}$ signals grow slowly and then plateau. To take the change 
in electron censity into account, considerctie following sodel for temission of photons fron inwazc diffusing ises in steady state plasma. It has beer previously otser;ejizat cact jorization sate, $i$, oniy radiates from a cylindrical shell lof width $(W)$ in the plasma. The emitted photon signal per unit area is proportional to the number/area, $N_{i}$, of those ions in that shel1, and to the nuinber/area, Ne, of electrons in that shell. If there is a unit area source for these ions of strength $S$, then the number of emitted photons/area is $n_{p}=N_{i} B R N_{e}=S W B R N_{e} / V$. where $B$ is the branching ratio, $V$ is the dxitt velocity of the ions ehrough the shell, and $A$ is the excitation rate. Now, the width of the shell depends on the innization rate, $Y_{i}$, the electron density and the drift velocity in the following fashion: weV/n ${ }_{i}{ }_{i}$. The number of emitted photons is then,

$$
n_{p}=R N_{e} S W B / V=S B R V / Y_{i} n_{e}
$$

For neoclassical ion transport, as observed in $\operatorname{ATC}^{2}{ }^{3} v$ is proporcional to $n_{e}$. hence $n_{p}$ is independant of $n_{e}$. Thus both the $T i$ and Fe signals indicate slow (- $5 \mathrm{~ms}$ ) increases in the influx rate of these atoms. Similarly, the 0 and $C$ signals indicate at first step-like increases and then linearly increasing influxes. (Between the beginning and the end of the 70 high power neutral beam discharges the OVI signal decreased by nearly a factor of two.)

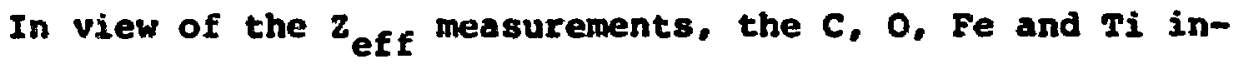
fluxes can, in no way, account for the factor of two increase in the electron density. That must come from an influx of $H$ or $D$. The behavior of the $F e, T i$ and $C$ signals after compression ( $t=30 \mathrm{~ms}$ ) is complex. It may be complicated by shot to shot 
plasma motion into and out of the line of sight of the vuv monochrometer. The behavior of the ovI emission is not complicated by the compression. That the ovI signal dces not instantaneously decrease after the neutral beam is shut off is important evidence that the bulk of the oxygen influx is not due to wall bombardment by the escaping beam $\mathrm{CX}$ particles.

\section{PLASMA-WALI INTERACTION}

Using in situ Auger electron spectroscopy, surface analysis of a gold and aluminum plated stainless steel sample, which had been positioned $20 \mathrm{~cm}$ from the plasma during 70 high power discharges, was performed. The details are reported elsewhere. The major observations were: the gold and aluminum plated sample became coated with monolayer coverage of hydrocarbons, carbon monoxide and water during its several hours of exposure to ATC vacuum prior to the beam-heated discharges; after exposure to the discharges the $C$ Auger signal increased by 208 , the 0 signal decreased by 508, and the All and Al signals disappeared; trace amounts of $\mathrm{S}$ and $\mathrm{Cl}$ appeared; no Fe or $\mathrm{Ti}$ was detected.

Qualitatively, the decrease of 0 on the sample is consistent with both the presence of $O$ in the discharge and the decrease with shot number of 0 in the discharge. After the termination of a discharge the free $O$ can be effectively pumped away by the $T i$ gettering. In contrast, the increase of $C$ and disappearance of Al and Au on the sample surface indicais st that hydrocarbon residual gases were readily adsorbed on the bombarded region of the sample and that they were not pumped by the Ti gettering. 
Fluxes to the ATC wall and 1 imiter, and semi-quantitative estimates of the resultant impurity influx rates during the beamheated stage of these discharges are presented in Table 2 . Though the fluxes out of the plasma are known to better than 508 accuracy, the efficiencies of sputtering or desorption are uncertain by about an order of magnitude. This is due to lack of basic surface measurements and to uncertainties about the surface conditions on the entire ATC vacuum vessal. However, the VuV signals (Figure 5) can be used to check the accuracy of Table 2. Consider the OVI signal. The step-like increase at $18 \mathrm{~ms}$, when the s.eutral beams are first turned on, indicates a rapid change of some flux to the wall. The obvious candidate for this is the beam $C x$ flux. Previously we noted that the 0 coricentration at $26 \mathrm{~ms}$ was of order 38 . Hence the step increase in the OVI signal should correspond to an additional 0.580 concentration at the and of the discharge. Iine 5 in Table 2 is within 25 of this. The increasing $C$ and $O$ signals from 18 to $28 \mathrm{~ms}$ are in step with the increasing flux and mean energy of the escaping plasma CX neutrals. When the beam is shut off at $28 \mathrm{~ms}$ the ovI signal does not instantaneously revert to its pre-injection value. Instead it slowly decreases in the same fashion as the plasma ion temperature. Accordingly, we identify the cause of this 0 and $C$ influx to be escaping plasma $C x$ neutrals. The value of $Z_{\text {eff }}$ at $26 \mathrm{~ms}$ indicates a 380 concentration. This is within 25\% of the value on line 7 in Table 2 . So all the $c$ and 0 influx can be attributed to $c x$ neutrals. Because 0 is more prevalent in the discharge than $\mathrm{C}$, more $\mathrm{H}_{2} \mathrm{O}$ than $\mathrm{CO}$ or $\mathrm{HC}^{\prime} \mathrm{s}$ is being desorbed. 
The Fe and $T i$ signals show: saturation between 18 and $28 \mathrm{~ms}$. The ion temperature shows a similar trend (cf. Figure 2 ). This would indicate that plasma $C X$ sputtering (line 8) was responsible lor the Fe and $T i$ influxes. From line 8 Table 2 we see that the estimated Fe influx due to plasma CX sputtering is even of the right magnitude. (DeMarcd has previously shown that the Fe concentration in ATC was approximately 0.18 .1 llowever there are three complications to this interpretation. Firstly, there is another mechanism that might cause increased Fe influx. The thermal load on the limiter exceeas $150 \mathrm{~kW}$. Depending on the spatial distribution of the load. either evaporation or fragmentation' occur. Secondly, from line 6 Table 2, we should expect a similar incre. ae in the $F e$ influx due to bean $C X$ losses. The predicted step is not evident in Figire 5a,b. Finally, if the entire ATC vacuum vessal wall is covered with hvdrocarbons to the same extent as the sample surface, it would be doubtful that Fe atoms could be ejected from the coated walls. Thus we cannot say that Cx sputtering causes the main influx of Fe into ATC.

The effects of these impurities on the beam-heated discharges are:

1) to increase the Ohmic heating efficacy (the ion-electron couplingl, thereby increasing both the electron ard ion temperatures. A higher electron temperature decreases the beam energy losses to the electrons.

2) to increase the electron density by about $20 \%$. This increases the energy confinement time.

3) to increase the radiation losses to $30 \mathrm{~kW}$ (158 of the OH input).

4) to speed up the beam plasma equil ibrium. This decreases 
the loss of energy by beam $C x$ by about 108. Also, the plasma size and density are sufficiently small that these impurities do not significantly reduce the penetration of the neutral beams.

From the above it is clear that these small amounts of impurities have a net beneficial effert during high power beam-heated discharges.

It is a pleasure to acknowledge useful discussions with J. Cecchi, C. Daughney, H. Eubank, H.Hsuan, and E. Marmar. 


\section{REFERENCES}

* Work supported sy U.S. Energy Research and Development Administration Contract E(11-L)-3073.

1. For a review of these effects see S.A.Cohen, J. vac. Sci. and Tech, 13, (1976).

2. R,A.Ellis, H.P.Eubark, R.Goldston, R.R.Smith, and T. Nagashima, Matt 1202 , (1976).

2. S,A.Cohen and H.F.Dylla, Matt 1227, 11976), (submitted to Nucl,Fusion).

4. H.Hsuan, K.Bol, and R.A.Ellis, Nucl. Fusion, 15, 657, (1976).

5. C. C.Daughney (to be published in Nucl. Fusion).

6. The physical cortent in this computer code is described in. D.M.Meade, et al, Proceedings of the Fifth International Conference on Plasma Phycics and Controlled Thermonuclear Fusion Research, Tokyo, Japan, 1974 (IAEA, Vienna, Austria, 1975) Paper No.

7. The power lost to the walls by this edge plasma, $n_{e} t_{t}^{A / 4}$, cannot exceed 58 of the Ohmic heating power input, where $v_{t}$ is the thermal velocity $=10^{8} \mathrm{~cm} / \mathrm{sec}$ and $\mathrm{A}$ is the surface area of the ATC plasma $=6 \times 10^{4} \mathrm{~cm}^{2}$.

8. P.E.Stott, C.C.Daughney and R.A.Ellis, Matt 1111 (1975), isubmitted to Nucl. Fusion).

9. H.P. Eubank( private communication).

10. H. Verbeek, ORNL 75-66 (submitted for publication).

11. H. Hsuan, (private comnunication).

12. J. L. Cecchi, Bull. Am. Phys. Soc., 19, 853, (1974). 
$-12-$

13. 3. A. Cohen, J. L. Cecchi and E. S. Marmar, Phys. Rev. Lett., 35. 1507, (1975).

i4. J. L. Cecchi (private commurication).

15. T. H. Stix, Plasma Physics 14, 367, (1972).

16. F. DeMarco, hatt 1012, (1973).

17. J. F. SchiveIl and D. J. Grove, Surface Effects in Controlled Pusion, editeà by $R$. Weidersich, M. Kaminsky and $C$. Zwilsky, (North iolland Publisning Co., Amsterdam, 1974), p. 107. 


\section{Figure Captions}

Fig. 1. Calculated electron and ion density and temperature profiles. These values are for $8 \mathrm{~ms}$ after the start of $230 \mathrm{~kW}$ of neutral beam heating. Experiment measurements show $15 \%$ higher ion temperature, 158 lower electron temperature and an axially peaked density.

Fig. 2. Measured time evolution of the central ion temperature and average electron density during a high power $(230 \mathrm{kWT})$ beam-heated ATC discharge. The surface power loss measured during low power $(90 \mathrm{~kW})$ beam-heated discharges is also shown.

Fig. 3. Calculated e nergy balance in bieam-heated ATC. The electron and ion heat conduction terms (-•-.--) represent the heat load to the limiter. The beam charge exchange, plasma charge exchange, and radiation terms (-) represent the surface heat load. The calculated electron density and ion temperature are also shown.

Fig. 4. Calculated flux of charge exchange neutrals from ATC for high power beam-heated discharge. The triangular peaks at $E=7.5$ and $15 \mathrm{keV}$ represent the escaping half and full energy beam charge exchange neutrals. The greatest number of escaping charge exchange neutrals have energies below $1 \mathrm{keV}$ and originate from the bulk plasma. 
Fig. 5. Measured ultraviolet radiation from selected ions of $\mathrm{Fe}, \mathrm{Ti}, \mathrm{C}$ and $\mathrm{O}$ during high power neutral beam heating. The ionization potentials for the observed states are noted to the right of each state. For the Fe, $\mathrm{C}$, and Ti data the plasma was compressed at $t \simeq 30 \mathrm{~ms}$. The plasma was not compressed during observations of the $O V I$ line. 
Table 1

Measured ATC Parameters at Different Times During a High Power Deuterium Neutral Beam Heated Deuterium Discharge

$\begin{array}{ccc} & \text { Ohmic } & \text { Beam Heating Compressional } \\ \text { Parameter Heating Alone } & \text { of } 230 \mathrm{~kW} \text { Heating }(C=2.1)\end{array}$

1. Time (ms)

2. Time of Measurements (ms)

3. Major Radius (cm)

4. Minor Radius $(\mathrm{cm})$

5. Magnetic Field ( $k G)$

6. Plasma Current (kA)

7. Loop Voltage

8. Central Electron Temperature (ev)

9. Edge Electron 14 Temperature (eV)

10. Central Eleç̧̧on Density $\left(\mathrm{cm}^{-3}\right)$ 11. Edge Electron,

12. Central Ion Temperature (ev)

13. Central Neuţ̧al Density $\left(\mathrm{cm}^{-3}\right)$

14. Ion Energy Confinement Time (ms)

15. ${ }^{2}$ eff

16. Neutron
FIux $\left(\mathrm{cm}^{-2} \mathrm{~s}^{-1}\right)$

$$
18-28
$$$$
30-45
$$

\section{5}

88

17

20

66

2.4

$800^{1}$

$10^{+}$

$2 \times 10^{13}$

$10^{10 *}$

200

$4.5 \times 10^{8 * *}$

$1.2^{\dagger}$

$<30$

\section{6}

88

17

20

66

2.4

850

$10^{\dagger}$

$3.9 \times 10^{13}$

$$
10^{10 *}
$$

420
1220

34

42

12

42

140

2.4

$1700^{\dagger}$

$$
10^{+}
$$

$1.1 \times 10^{14}$

$$
10^{10 *}
$$

10
$2 \times 10^{8 * * *}$

$$
3.2
$$

3.2

$3 \times 10^{5}$

* Estimated density $1 \mathrm{~cm}$ from the vacuum vessel wall.

* Taken from the computer simulation. These values agree reasonably well $( \pm 50 \%)$ with previous measurements on similar discharges.

*** Estimate based on ionization and charge exchange depletion of the central neutral density.

+ Measured for similar discharges. 
Table 2

Rate of Desorption or Sputtering of Atoms from the Wall of ATC during Beam Injection

\section{Flux to the Wall}

Energy

Particle $\begin{gathered}\text { Number } \\ \left(\mathrm{cm}^{-2} \mathrm{sec}^{-1}\right)\end{gathered}$

(iv $/ \mathrm{cm}^{2} \mathrm{sec}$

$5 \times 10^{4} \quad 2 \times 10^{-9}$

S

.003

$10^{13}$

D

1.5

D

$10^{-4}$

$<.1$

D

$10^{-4}$

$4 \times 10^{-4}$

Edge Plasma

(10 ev)

5. Beam CX

Neutrals

(15 keV D ${ }^{\circ}$ )

6.

7. Plasma CX

Neutrals

$(50-1000 \mathrm{eV})$

8.

9. Plasma

Thermal

Load on

Limiter

\section{Flux to the Plasma}

$2 \times 10^{-8}$

$5 \times 10^{-4}$

$$
2 \times 10^{17}
$$

$$
10^{15}
$$

1

$D$

$10^{-1}$

$2 \times 10^{-3}$

$10^{-2}$

"

S

$10^{-1}$

$2 \times 10^{-2}$

$10^{16}$

.1

D

$10^{-3}$

$2 \times 10^{-4}$

$\mathbf{S}$

$\sim 10^{3}$
Resultant

Change in

Efficiency wall coverage impurity concentration in discharge (o) Species

$2 \times 10^{-14}$ $\mathrm{O}, \mathrm{Fe}$

$$
10^{-16}
$$

$4 \times 10^{-6}$

$0, \mathrm{C}$

0.1

$0, \mathrm{C}$

$2 \times 10^{-4}$

.4

$0, c$

.04

Fe, Ti

4.

$0, c$

.04

Fe,Ti 


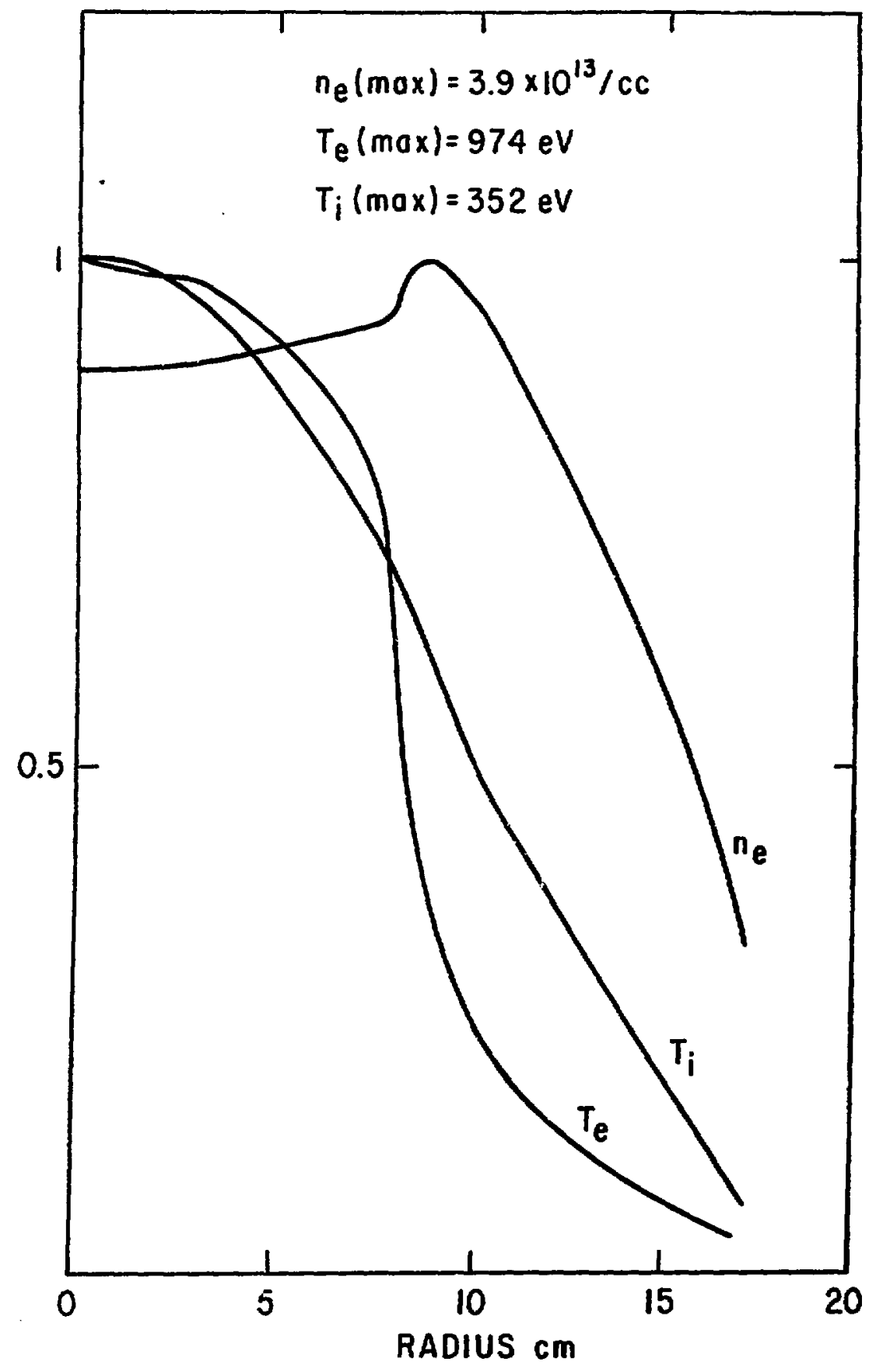




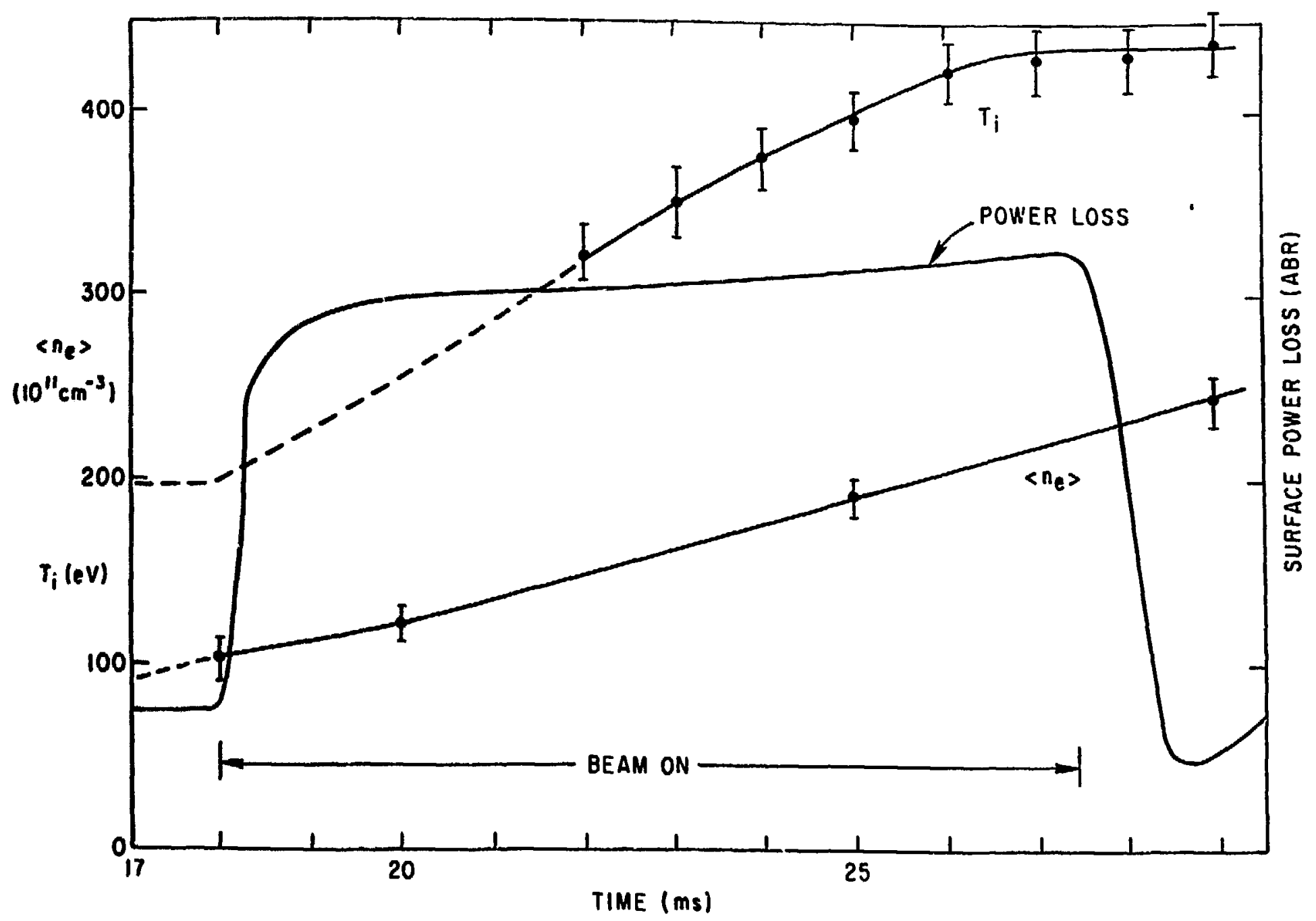




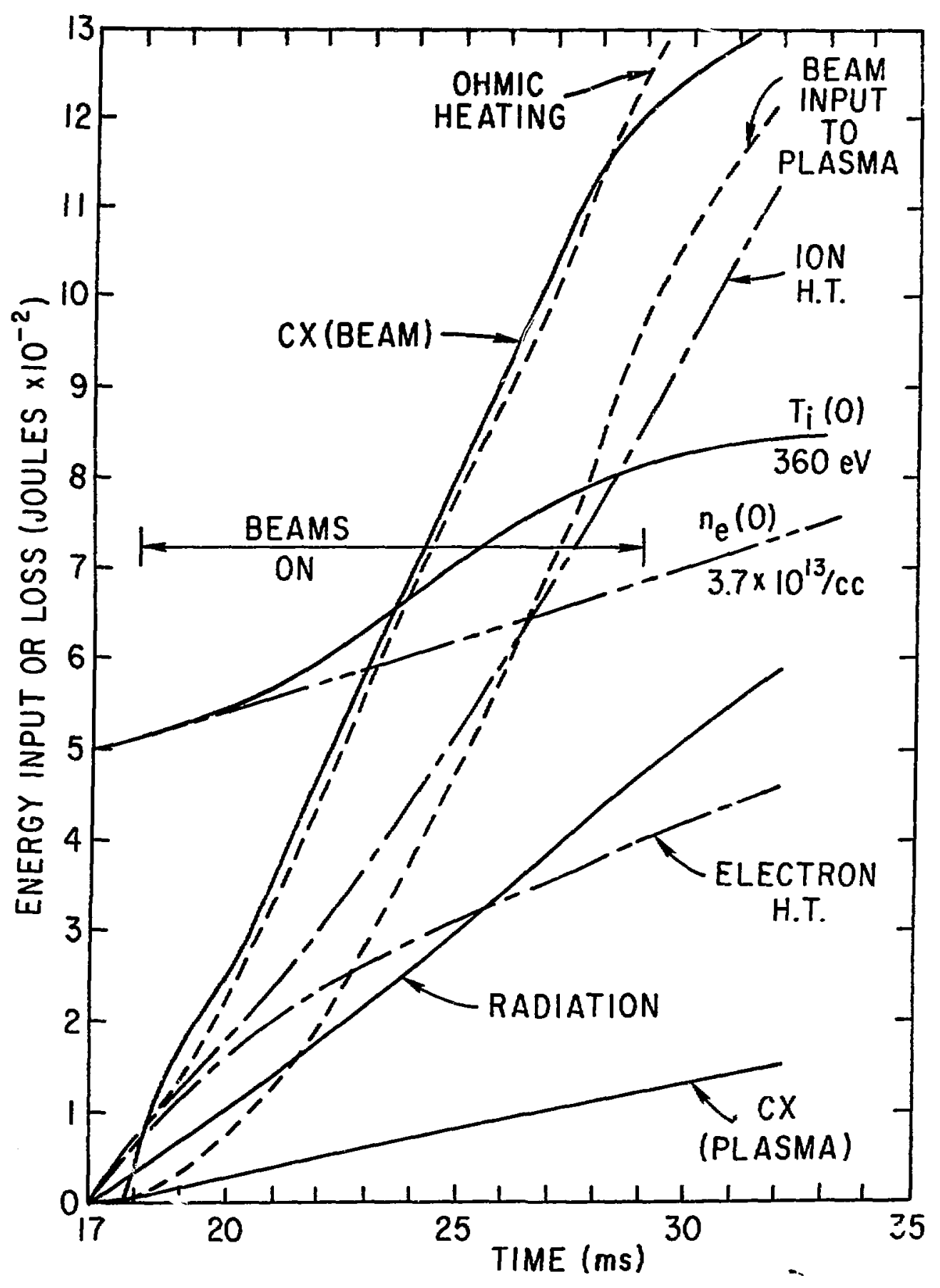




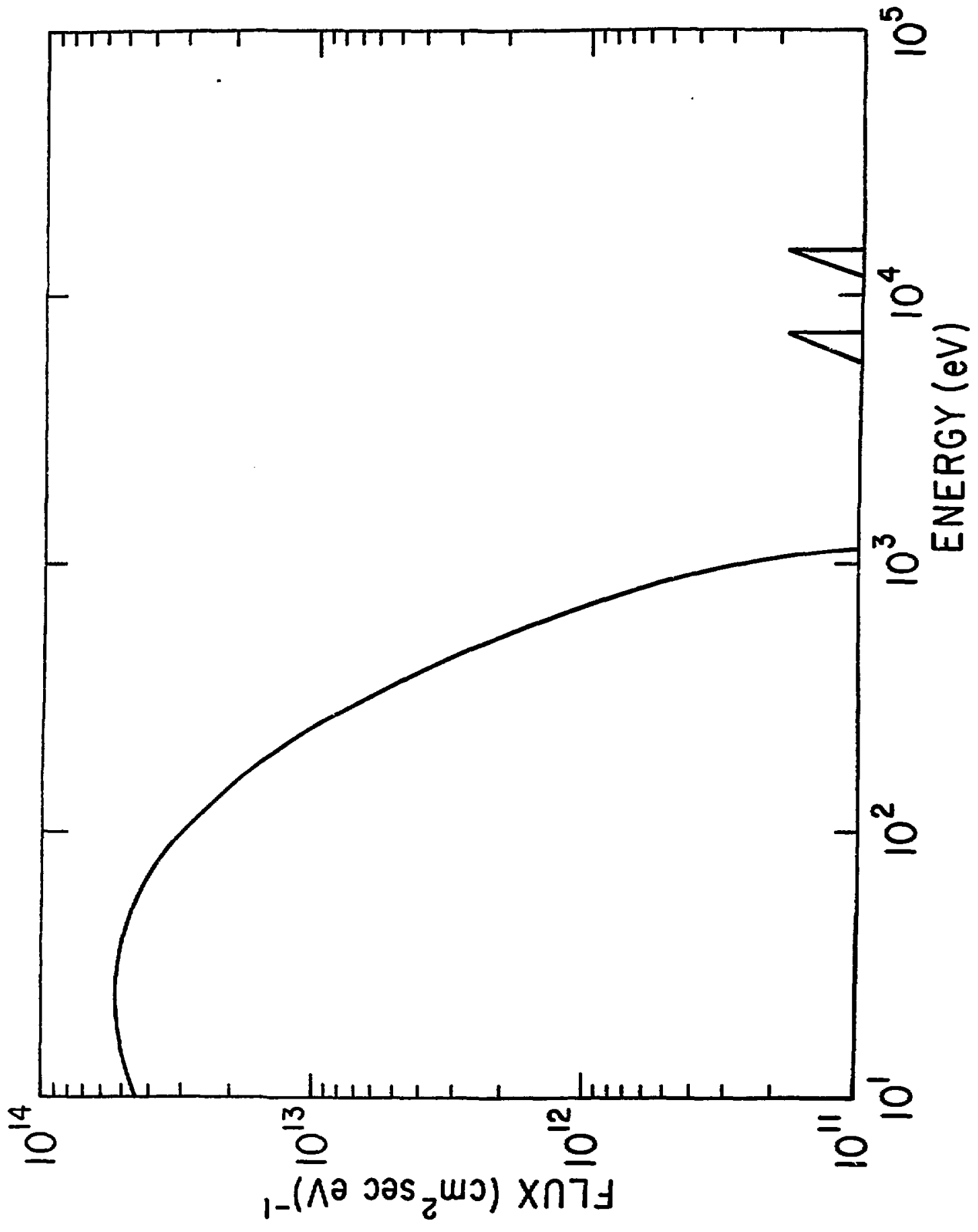




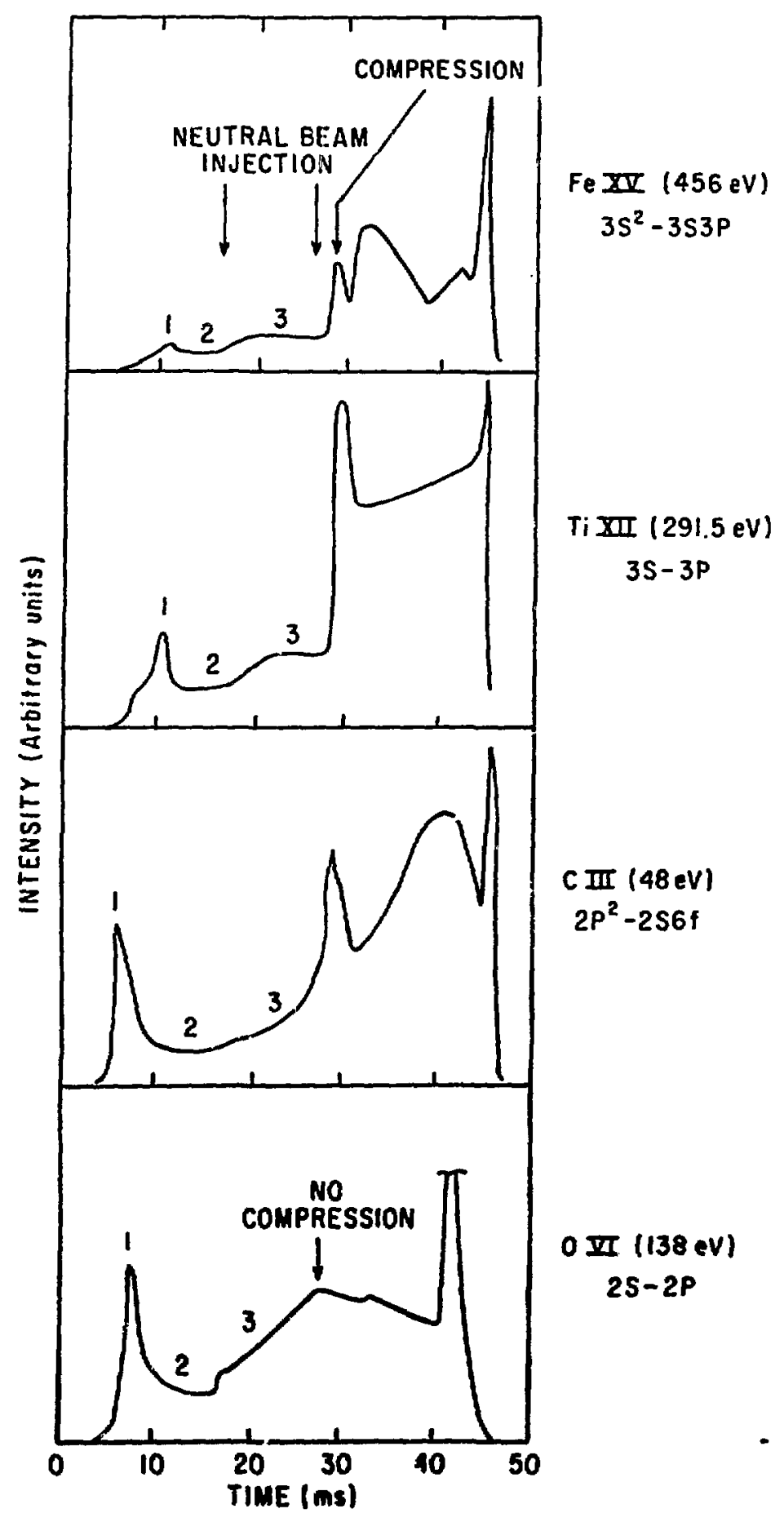

